\title{
Bohnenblust-Hille inequalities: analytical and computational aspects
}

\author{
WASTHENNY V. CAVALCANTE ${ }^{1}$ and DANIEL M. PELLEGRINO ${ }^{2}$ \\ ${ }^{1}$ Universidade Federal de Pernambuco/UFPE, Av. Jornalista Aníbal Fernandes, s/n, Cidade Universitária, \\ Departamento de Matemática, 50740-560 Recife, PE, Brazil \\ ${ }^{2}$ Universidade Federal da Paraíba/UFPB, CCEN, Departamento de Matemática, \\ Cidade Universitária, 58051-900 João Pessoa, PB, Brazil
}

Manuscript received on May 25, 2017; accepted for publication on June 29, 2017

\begin{abstract}
How to cite: CAVALCANTE WV AND PELLEGRINO DM. 2019. Bohnenblust-Hille inequalities: analytical and computational aspects. An Acad Bras Cienc 91: e20170398. DOI 10.1590/0001-3765201720170398.

Abstract: The Bohnenblust-Hille polynomial and multilinear inequalities were proved in 1931 and the determination of exact values of their constants is still an open and challenging problem, pursued by various authors. The present paper briefly surveys recent attempts to attack/solve this problem; it also presents new results, like connections with classical results of the linear theory of absolutely summing operators, and new perspectives.
\end{abstract}

Key words: Bohnenblust-Hille inequality, Hardy-Littlewood inequality, extreme points, Banach spaces.

\section{INTRODUCTION}

Motivated by a question raised by P.J. Daniell concerning the existence of certain functions of bounded variation, Littlewood (1930) proved that

$$
\left(\sum_{i, j=1}^{n}\left|T\left(e_{i}, e_{j}\right)\right|^{\frac{4}{3}}\right)^{\frac{3}{4}} \leq \sqrt{2} \sup _{\|x\|,\|y\| \leq 1}|T(x, y)|
$$

for all bilinear forms $T: \ell_{\infty}^{n} \times \ell_{\infty}^{n} \rightarrow \mathbb{C}$ and all positive integers $n$ (as usual, $\ell_{\infty}^{n}$ denotes $\mathbb{C}^{n}$ with the sup norm and $e_{j}$ its canonical unit vectors). This inequality is now called Littlewood's $4 / 3$ inequality. One year later, Bohnenblust and Hille (1931) extended Littlewood's 4/3 inequality to the multilinear framework by

2010 Mathematics Subject Classification: 46B20, 46G25, 52B11

Correspondence to: Daniel Marinho Pellegrino

E-mail: pellegrino@pq.cnpq.br / dmpellegrino@gmail.com

ORCid: http://orcid.org/0000-0002-5369-5630

* Contribution to the centenary of the Brazilian Academy of Sciences. 
proving a key result to solve a long standing problem posed by H. Bohr (1913), related to the convergence of Dirichlet series. Bohnenblust and Hille proved that the optimal constants $B_{\mathbb{C}, m}(n) \geq 1$ satisfying

$$
\left(\sum_{j_{1}, \ldots, j_{m}=1}^{n}\left|T\left(e_{j_{1}}, \ldots, e_{j_{m}}\right)\right|^{\frac{2 m}{m+1}}\right)^{\frac{m+1}{2 m}} \leq B_{\mathbb{C}, m}(n)\|T\|,
$$

for all $m$-linear forms $T: \ell_{\infty}^{n} \times \cdots \times \ell_{\infty}^{n} \rightarrow \mathbb{C}$ are such that

$$
B_{\mathbb{C}, m}:=\sup _{n} B_{\mathbb{C}, m}(n)<\infty .
$$

Above and henceforth, as usual,

$$
\|T\|:=\sup _{\left\|x^{(1)}\right\|, \ldots,\left\|x^{(m)}\right\| \leq 1}\left|T\left(x^{(1)}, \ldots, x^{(m)}\right)\right| .
$$

The case $m=2$ in (1) recovers Littlewood's $4 / 3$ inequality. As a matter of fact Bohnenblust and Hille seemed to be more interested in the following variant of (1): there is a constant $C_{\mathbb{C}, m} \geq 1$ such that

$$
\left(\sum_{|\alpha|=m}\left|c_{\alpha}(P)\right|^{\frac{2 m}{m+1}}\right)^{\frac{m+1}{2 m}} \leq C_{\mathbb{C}, m} \sup _{\|x\| \leq 1}|P(x)|
$$

for all $m$-homogeneous polynomials $P: \ell_{\infty}^{n} \rightarrow \mathbb{C}$ of the form $P(x)=\sum_{|\alpha|=m} c_{\alpha}(P) \mathbf{x}^{\alpha}$. It is worth mentioning that the exponent $2 m /(m+1)$ in (1) and (2) is sharp.

Both multilinear and polynomial inequalities also hold for real scalars instead of complex scalars. From now on $\mathbb{K}$ will denote $\mathbb{R}$ or $\mathbb{C}$. The case of complex scalars is the original one, with strong connections with Analytic Number Theory and Dirichlet series; we mention Defant et al. (2011) and Bayart et al. (2014) for this line of research. The case of real scalars was just very recently explored but seems to be also very relevant as it can be seen in its applications in Quantum Information Theory, remarked by Montanaro (2012). It may sound surprising but, by now, the estimates of the constants are what really matters for the applications.

The original estimates obtained by Bohnenblust and Hille were

$$
\begin{aligned}
& 1 \leq B_{\mathbb{C}, m} \leq m^{\frac{m+1}{2 m}}(\sqrt{2})^{m-1}, \\
& 1 \leq C_{\mathbb{C}, m} \leq B_{\mathbb{C}, m} \frac{m^{\frac{m}{2}}(m+1)^{\frac{m+1}{2}}}{2^{m}(m !)^{\frac{m+1}{2 m}}}
\end{aligned}
$$

and since 2011 a series of papers have been dedicated to the investigation of the constants $B_{\mathbb{C}, m}, C_{\mathbb{C}, m}, B_{\mathbb{R}, m}$, $C_{\mathbb{R}, m}$. Up to now, according to Bayart et al. (2014) and Nuñez-Alarcón (2013), the best known asymptotic estimates for these constants in the case of complex scalars are

$$
\left\{\begin{array}{c}
1 \leq B_{\mathbb{C}, m} \leq m^{\frac{1-\gamma}{2}}<m^{0.212} \\
\left(1+2^{1-m}\right)^{\frac{m-1}{4 m}} \leq C_{\mathbb{C}, m} \leq \kappa(1+\varepsilon)^{m}
\end{array}\right.
$$

where $\gamma$ is the Euler-Mascheroni constant, $\varepsilon>0$ can be arbitrarily chosen and $\kappa$ depends on $\varepsilon$. It has been recently shown in Maia et al. (2017) that under certain mild assumptions, $\left(C_{\mathbb{C}, m}\right)_{m=1}^{\infty}$ is bounded. For real 
scalars, combining information from Bayart et al. (2014), Campos et al. (2015) and Diniz et al. (2014) we have

$$
\left\{\begin{array}{c}
2^{1-\frac{1}{m}} \leq B_{\mathbb{R}, m} \leq 2^{\frac{446381}{55440}-\frac{m}{2}} \prod_{j=14}^{m}\left(\frac{\Gamma\left(\frac{3}{2}-\frac{1}{j}\right)}{\sqrt{\pi}}\right)^{\frac{j}{2-2 j}}<1.3 m^{0.365}(\text { for } m \geq 14) \\
(1.17)^{m}<C_{\mathbb{R}, m} \leq(2+\varepsilon)^{m},
\end{array}\right.
$$

for some (small) $\varepsilon>0$.

Despite the huge recent advances in the theory (exponential estimates were improved to sublinear estimates and super-exponential estimates were improved to subpolynomial estimates), there are still many basic/simple open problems and new tools seem to be needed to a better understanding of the whole scenery. Below we list some basic open problems in this setting:

- Is the sequence $\left(B_{\mathbb{K}, m}\right)_{m=1}^{\infty}$ bounded? This was conjectured to be true in Pellegrino and Teixeira (2017) but it seems to be far from being solved.

- Is the sequence $\left(B_{\mathbb{K}, m}\right)_{m=1}^{\infty}$ increasing?

- Is $B_{\mathbb{C}, m}=1$ for all positive integers $m$ ?

- What is the asymptotic growth of $\left(C_{\mathbb{R}, m}\right)_{m=1}^{\infty}$ ?

Of course, a more ambitious problem is:

- What are the optimal values of $B_{\mathbb{K}, m}$ and $C_{\mathbb{K}, m}$ ?

One can also try to figure out a complete perspective by attacking the even more ambitious question in the multilinear setting:

- For each positive integers $m, n \geq 2$, what are the optimal values $B_{\mathbb{K}, m}(n)$ ?

Hardy and Littlewood (1934) extended Littlewood's $4 / 3$ inequality to bilinear forms on $\ell_{p}^{n}$ spaces. The multilinear version of their result was obtained in Praciano-Pereira (1981) for $4 \leq 2 m \leq p \leq \infty$. The result is summarized as follows: there exists a constant $C_{\mathbb{K}, m, p} \geq 1$ such that, for all continuous $m$-linear forms $T: \ell_{p}^{n} \times \cdots \times \ell_{p}^{n} \rightarrow \mathbb{K}$, and all positive integers $n$,

$$
\left(\sum_{j_{1}, \ldots, j_{m}=1}^{n} \mid T\left(e_{j_{1}}, \ldots, e_{j_{m}}\right)^{\frac{2 m p}{m p+p-2 m}}\right)^{\frac{m p+p-2 m}{2 m p}} \leq C_{\mathbb{K}, m, p}\|T\|,
$$

and the exponent $\frac{2 m p}{m p+p-2 m}$ is optimal. Further generalizations to the anisotropic settings were obtained in Albuquerque et al. (2014) and the best known estimates for $C_{\mathbb{K}, m, p}$ can be found in Araujo and Pellegrino (2014, 2017) and Cavalcante et al. (2016). The case $m<p<2 m$ was recently explored in Dimant and Sevilla-Peris (2016) and the constants involved were further explored in Albuquerque et al. (2017), Nunes (2017), among others.

The main goal of this paper is to survey some aspects of the Bohnenblust-Hille and Hardy-Littlewood inequalities and also present new results and perspectives. These inequalities have been exhaustively investigated in the recent years and several new results and new techniques have appeared. We are also interested 
in showing connections between these inequalities and some classical results of the theory of absolutely summing operators.

This paper is organized as follows. In the second section we show how the investigation of the geometry of the unit ball of the spaces of multilinear forms can be potentially useful to reach the optimal constants. In the third section we show how these kind of inequalities recover classical results of the linear theory of absolutely summing operators. In the final section we discuss the perspectives of the subject and new strategies to attack the problem.

\section{THE MULTILINEAR BOHNENBLUST-HILLE CONSTANTS: GEOMETRIC APPROACHES}

In this section we discuss how the geometry of the unit balls of Banach spaces is connected to the problem of finding optimal constants of the multilinear Bohnenblust-Hille inequality.

\section{ESTIMATING NORMS}

Given an $m$-linear form $T: \ell_{\infty}^{n} \times \cdots \times \ell_{\infty}^{n} \rightarrow \mathbb{K}$ is there a handy formula for $\|T\|$ ? If the answer was positive, then a definitive answer to the optimization problem of finding the optimal constants of the BohnenblustHille inequality could be eventually achieved by a kind of Lagrange Multipliers approach. Unfortunately, to estimate the norm of a general multilinear form seems to be a quite complicated task. The following result (of independent interest) is a prediction:

Proposition 1. Let $T: \ell_{\infty}^{2}(\mathbb{C}) \times \ell_{\infty}^{2}(\mathbb{C}) \rightarrow \mathbb{C}$ be given by $T(z, w)=\sum_{i, j=1}^{2} a_{i j} z_{i} w_{j}$ with $a_{i j} \in \mathbb{R}$. Then

$$
\begin{aligned}
& \|T\|=\max \left\{\begin{array}{c}
\left|a_{11}+a_{21}\right|+\left|a_{12}+a_{22}\right|,\left|a_{11}-a_{21}\right|+\left|a_{12}-a_{22}\right| \\
\quad \sqrt{a_{11}^{2}+a_{21}^{2}+2 a_{11} a_{21} \frac{\frac{a_{11}^{2} a_{21}^{2}}{a_{12}^{2} a_{22}^{2}}\left(a_{12}^{2}+a_{22}^{2}\right)-\left(a_{11}^{2}+a_{21}^{2}\right)}{2 a_{11} a_{21}\left(1-\frac{a_{11} a_{21}}{a_{12} a_{22}}\right)}} \\
+\sqrt{a_{12}^{2}+a_{22}^{2}+2 a_{12} a_{22} \frac{\frac{a_{11}^{2} a_{21}^{2}}{a_{12}^{2} a_{22}^{2}}\left(a_{12}^{2}+a_{22}^{2}\right)-\left(a_{11}^{2}+a_{21}^{2}\right)}{2 a_{11} a_{21}\left(1-\frac{\left.a_{11} a_{21}\right)}{a_{12} a_{22}}\right)}}
\end{array}\right\} \\
& \text { if }\left(a_{11}, a_{21}, a_{12}, a_{22}\right) \in(\mathbb{R} \backslash\{0\})^{4} \text { and } \operatorname{sgn}\left(\frac{a_{11} a_{21}}{a_{12} a_{22}}\right)=-1 \text { and } \\
& \left|\frac{a_{11}^{2} a_{21}^{2}}{a_{12}^{2} a_{22}^{2}}\left(a_{12}^{2}+a_{22}^{2}\right)-\left(a_{11}^{2}+a_{21}^{2}\right)\right| \leq\left|2 a_{11} a_{21}\left(1-\frac{a_{11} a_{21}}{a_{12} a_{22}}\right)\right| ;
\end{aligned}
$$

(B)

$$
\|T\|=\max \left\{\left|a_{11}+a_{21}\right|+\left|a_{12}+a_{22}\right|,\left|a_{11}-a_{21}\right|+\left|a_{12}-a_{22}\right|\right\}
$$

otherwise.

Proof. Note that

$$
\|T\|=\sup \left\{\left\|T_{z}\right\|:\|z\|_{\infty} \leq 1\right\},
$$

where $T_{z}: \ell_{\infty}^{2}(\mathbb{C}) \rightarrow \mathbb{C}$ is given by

$$
T_{z}(w)=\left(a_{11} z_{1}+a_{21} z_{2}\right) w_{1}+\left(a_{12} z_{1}+a_{22} z_{2}\right) w_{2} .
$$


We thus have

$$
\|T\|=\sup \left\{\left\|T_{z}\right\|:\|z\|_{\infty} \leq 1\right\}=\sup \left\{\left|a_{11} z_{1}+a_{21} z_{2}\right|+\left|a_{12} z_{1}+a_{22} z_{2}\right|:\|z\|_{\infty} \leq 1\right\} .
$$

Hence, calculating $\|T\|$ is the same of maximizing the function

$$
f\left(z_{1}, z_{2}\right)=\left|a_{11} z_{1}+a_{21} z_{2}\right|+\left|a_{12} z_{1}+a_{22} z_{2}\right|
$$

on $B_{\ell_{\infty}^{2}(\mathbb{C})}:=\left\{\left(z_{1}, z_{2}\right) \in \ell_{\infty}^{2}(\mathbb{C}):\left\|\left(z_{1}, z_{2}\right)\right\|_{\infty} \leq 1\right\}$. Since $f$ is a convex function on $B_{\ell_{\infty}^{2}(\mathbb{C})}$, a consequence of the Krein-Milman Theorem assures that the maximum of $f$ is attained on some extreme point of $B_{\ell_{\infty}^{2}(\mathbb{C})}$ (this will be discussed in the depth in the next subsection). It is simple to verify that extreme points of $B_{\ell_{\infty}^{2}(\mathbb{C})}$ (this result is used, for instance, by Diestel et al. 1995) are of the form $\left(z_{1}, z_{2}\right)$ with $\left|z_{1}\right|=\left|z_{2}\right|=1$. Denoting $z_{j}=x_{j}+i y_{j}, j=1,2$, we have

$$
\begin{aligned}
f\left(z_{1}, z_{2}\right) & =\sqrt{\left(a_{11} x_{1}+a_{21} x_{2}\right)^{2}+\left(a_{11} y_{1}+a_{21} y_{2}\right)^{2}} \\
& +\sqrt{\left(a_{12} x_{1}+a_{22} x_{2}\right)^{2}+\left(a_{12} y_{1}+a_{22} y_{2}\right)^{2}} .
\end{aligned}
$$

Since $\left|z_{1}\right|=\left|z_{2}\right|=1$, we can write $z_{j}=\cos \theta_{j}+i \sin \theta_{j}, j=1,2$. Hence

$$
f\left(\theta_{1}, \theta_{2}\right)=\sqrt{a_{11}^{2}+a_{21}^{2}+2 a_{11} a_{21} \cos \left(\theta_{1}-\theta_{2}\right)}+\sqrt{a_{12}^{2}+a_{22}^{2}+2 a_{12} a_{22} \cos \left(\theta_{1}-\theta_{2}\right)} .
$$

By making $t=\theta_{1}-\theta_{2}$ we have

$$
f(t)=\sqrt{a_{11}^{2}+a_{21}^{2}+2 a_{11} a_{21} \cos t}+\sqrt{a_{12}^{2}+a_{22}^{2}+2 a_{12} a_{22} \cos t} .
$$

\section{- Proof of (A):}

We divide the proof of $(\mathrm{A})$ in two cases:

$\circ$ First case. Suppose that $\left(a_{11}, a_{21}, a_{12}, a_{22}\right) \in(\mathbb{R} \backslash\{0\})^{4}$ and $a_{11} \neq \pm a_{21}$ and $a_{12} \neq \pm a_{22}$.

In this case, since $\left(a_{11}, a_{21}, a_{12}, a_{22}\right) \in(\mathbb{R} \backslash\{0\})^{4}$ and $a_{11} \neq \pm a_{21}$ and $a_{12} \neq \pm a_{22}, f^{\prime}$ always exists and

$$
f^{\prime}(t)=\frac{-a_{11} a_{21} \sin t}{\sqrt{a_{11}^{2}+a_{21}^{2}+2 a_{11} a_{21} \cos t}}+\frac{-a_{12} a_{22} \sin t}{\sqrt{a_{12}^{2}+a_{22}^{2}+2 a_{12} a_{22} \cos t}}=0
$$

if, and only if, $t=k \pi, k \in \mathbb{Z}$ or

$$
\frac{-a_{11} a_{21}}{a_{12} a_{22}}=\frac{\sqrt{a_{11}^{2}+a_{21}^{2}+2 a_{11} a_{21} \cos t}}{\sqrt{a_{12}^{2}+a_{22}^{2}+2 a_{12} a_{22} \cos t}}
$$

Since $\operatorname{sgn}\left(\frac{a_{11} a_{21}}{a_{12} a_{22}}\right)=-1$, we have

$$
2 a_{11} a_{21}\left(1-\frac{a_{11} a_{21}}{a_{12} a_{22}}\right) \cos t=\frac{a_{11}^{2} a_{21}^{2}}{a_{12}^{2} a_{22}^{2}}\left(a_{12}^{2}+a_{22}^{2}\right)-\left(a_{11}^{2}+a_{21}^{2}\right)
$$

and since

$$
\left|\frac{a_{11}^{2} a_{21}^{2}}{a_{12}^{2} a_{22}^{2}}\left(a_{12}^{2}+a_{22}^{2}\right)-\left(a_{11}^{2}+a_{21}^{2}\right)\right| \leq\left|2 a_{11} a_{21}\left(1-\frac{a_{11} a_{21}}{a_{12} a_{22}}\right)\right|
$$


there is $t_{0}$ such that

$$
\cos t_{0}=\frac{\frac{a_{11}^{2} a_{21}^{2}}{a_{12}^{2} a_{22}^{2}}\left(a_{12}^{2}+a_{22}^{2}\right)-\left(a_{11}^{2}+a_{21}^{2}\right)}{2 a_{11} a_{21}\left(1-\frac{a_{11} a_{21}}{a_{12} a_{22}}\right)} .
$$

Thus

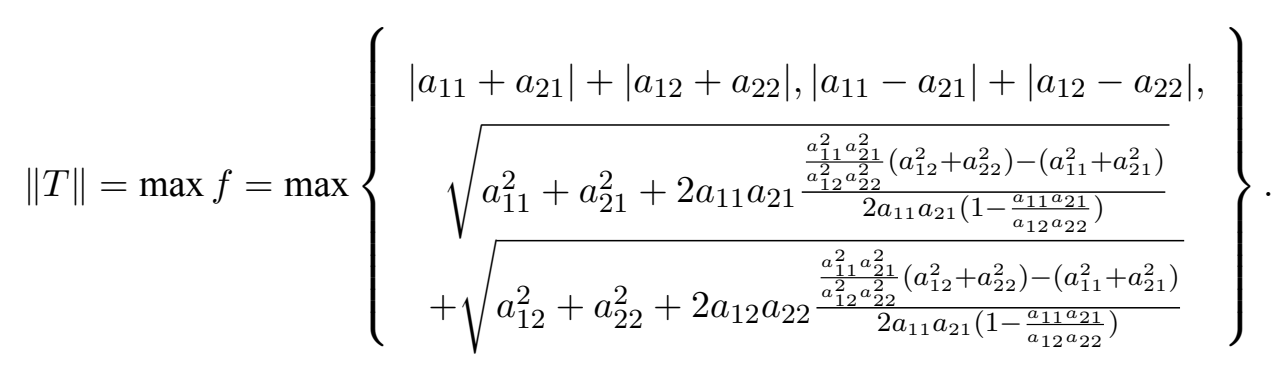

$\circ$ Second case. Suppose that $\left(a_{11}, a_{21}, a_{12}, a_{22}\right) \in(\mathbb{R} \backslash\{0\})^{4}$ and $\left(a_{11}= \pm a_{21}\right.$ or $\left.a_{12}= \pm a_{22}\right)$.

In this case there are real numbers $t_{0}$ such that $f^{\prime}\left(t_{0}\right)$ does not exist. For these values of $t_{0}$ we can see that

$$
f\left(t_{0}\right)=\left|a_{11}+a_{21}\right|+\left|a_{12}+a_{22}\right|
$$

or

$$
f\left(t_{0}\right)=\left|a_{11}-a_{21}\right|+\left|a_{12}-a_{22}\right| .
$$

For the values of $t$ such that $f^{\prime}(t)$ exists, we proceed as in the first case; therefore we also obtain (6).

- Proof of (B).

We consider three cases:

$\circ$ Case 1. Suppose $\left(a_{11}, a_{21}, a_{12}, a_{22}\right) \in(\mathbb{R} \backslash\{0\})^{4}, a_{11} \neq \pm a_{21}$ and $a_{12} \neq \pm a_{22}$ with

$$
\operatorname{sgn}\left(\frac{a_{11} a_{21}}{a_{12} a_{22}}\right)=1 \text {. }
$$

From (4) we can observe that $f^{\prime}(t)=0$ if and only if $t=k \pi, k \in \mathbb{Z}$ and thus

$$
\|T\|=\max f=\max \left\{\left|a_{11}+a_{21}\right|+\left|a_{12}+a_{22}\right|,\left|a_{11}-a_{21}\right|+\left|a_{12}-a_{22}\right|\right\} .
$$

$\circ$ Case 2. Suppose $\left(a_{11}, a_{21}, a_{12}, a_{22}\right) \in(\mathbb{R} \backslash\{0\})^{4}, a_{11} \neq \pm a_{21}, a_{12} \neq \pm a_{22}$,

$$
\operatorname{sgn}\left(\frac{a_{11} a_{21}}{a_{12} a_{22}}\right)=-1
$$

and

$$
\left|\frac{a_{11}^{2} a_{21}^{2}}{a_{12}^{2} a_{22}^{2}}\left(a_{12}^{2}+a_{22}^{2}\right)-\left(a_{11}^{2}+a_{21}^{2}\right)\right|>\left|2 a_{11} a_{21}\left(1-\frac{a_{11} a_{21}}{a_{12} a_{22}}\right)\right| .
$$

In this case, from (5) we also know that $f^{\prime}(t)=0$ if and only if $t=k \pi, k \in \mathbb{Z}$; therefore

$$
\|T\|=\max f=\max \left\{\left|a_{11}+a_{21}\right|+\left|a_{12}+a_{22}\right|,\left|a_{11}-a_{21}\right|+\left|a_{12}-a_{22}\right|\right\} .
$$

$\circ$ Case 3. We may have one of the following situations:

(1) $a_{11} a_{21}=0$ and $a_{12} a_{22}=0$; 
(2) $a_{11} a_{21}=0$ and $a_{12} a_{22} \neq 0$;

(3) $a_{11} a_{21} \neq 0$ and $a_{12} a_{22}=0$;

(4) $a_{11} a_{21} \neq 0$ and $a_{12} a_{22} \neq 0$.

If we consider (1), $f$ can be written as one of the following expressions:

(a) $f(t)=\left|a_{11}\right|+\left|a_{12}\right|$;

(b) $f(t)=\left|a_{11}\right|+\left|a_{22}\right|$;

(c) $f(t)=\left|a_{21}\right|+\left|a_{12}\right|$;

(d) $f(t)=\left|a_{21}\right|+\left|a_{22}\right|$.

We thus can write, in any case,

$$
f(t)=\left|a_{11}+a_{21}\right|+\left|a_{12}+a_{22}\right|=\left|a_{11}-a_{21}\right|+\left|a_{12}-a_{22}\right|
$$

and, of course, we obtain the expression of (B).

If we consider (2) there is no loss of generality in supposing $a_{11}=0$. So, we get

$$
f(t)=\left|a_{21}\right|+\sqrt{a_{12}^{2}+a_{22}^{2}+2 a_{12} a_{22} \cos t}
$$

and we consider two subcases.

- Subcase 1. If $a_{12}=a_{22}$ or $a_{12}=-a_{22}$, then there is a $t_{0} \in \mathbb{R}$ such that $f^{\prime}\left(t_{0}\right)$ does not exist. In this case, it is plain that

$$
f\left(t_{0}\right)=\left|a_{21}\right| \leq\left|a_{11}+a_{21}\right|+\left|a_{12}+a_{22}\right| .
$$

For other values of $t$ we have

$$
f^{\prime}(t)=\frac{-a_{12} a_{22} \sin t}{\sqrt{a_{12}^{2}+a_{22}^{2}+2 a_{12} a_{22} \cos t}}
$$

and thus $f^{\prime}\left(t_{1}\right)=0$ if and only if $t_{1}=k \pi$ and $k \in \mathbb{Z}$. For these values of $t_{1}$ we have

$$
f\left(t_{1}\right)=\left|a_{21}\right|+\left|a_{12}+a_{22}\right|=\left|a_{11}+a_{21}\right|+\left|a_{12}+a_{22}\right|
$$

or

$$
f\left(t_{1}\right)=\left|a_{21}\right|+\left|a_{12}-a_{22}\right|=\left|a_{11}-a_{21}\right|+\left|a_{12}-a_{22}\right| .
$$

We thus have again the expression given in (B).

$\circ$ Subcase 2. If $a_{12} \neq a_{22}$ and $a_{12} \neq-a_{22}$, then $a_{12}^{2}+a_{22}^{2}+2 a_{12} a_{22} \cos t \neq 0$ for all $t$ and $f^{\prime}(t)$ exists for all $t$; thus we again obtain the expression of (B).

The situation (3) is similar to (2).

If we have (4) and ( $a_{11}= \pm a_{21}$ or $\left.a_{12}= \pm a_{22}\right)$ we proceed as in the second case of(A). If $a_{11} \neq \pm a_{21}$ and $a_{12} \neq \pm a_{22}$ we are encompassed by Case 1 or Case 2 of (B).

For real scalars, for the obvious reasons, the expression of the norm is less complicated; this result can be found in Jameson (1994):

Proposition 2. Let $T: \ell_{\infty}^{2}(\mathbb{R}) \times \ell_{\infty}^{2}(\mathbb{R}) \rightarrow \mathbb{R}$ be given by $T(x, y)=\sum_{i, j=1}^{2} a_{i j} x_{i} y_{j}$, with $a_{i j} \in \mathbb{R}$. Then

$$
\|T\|=\max \left\{\left|a_{11}+a_{21}\right|+\left|a_{12}+a_{22}\right|,\left|a_{11}-a_{21}\right|+\left|a_{12}-a_{22}\right|\right\} .
$$


The above expressions of the norms of bilinear forms are somewhat prohibitive for further investigations following this vein. As we will see in the next section, the investigation of the geometry of the closed unit balls of $\mathcal{L}\left({ }^{m} \ell_{\infty}^{n}(\mathbb{K})\right)$ is important for our goals and may overcome the difficulty of finding formulas for the norms of multilinear forms.

\section{EXTREME POINTS AND OPTIMIZATION}

Given a vector space $E$ and a convex set $A \subset E$, a vector $x \in A$ is called extreme point of $A$ when $y, z \in A$ with $x=\frac{1}{2}(y+z)$ implies $y=z=x$. Extreme points are important for optimization of continuous and convex functions for a simple reason: their maximum are attained in extreme points as we shall see below.

Let us first state the Minkowski/Krein-Milman Theorem. It asserts that given a convex and compact subset $K \subset \mathbb{R}^{n}$, then $K=\operatorname{conv}(\operatorname{ext}(K))$. Here $\operatorname{ext}(K)$ denotes the set of all extreme points of $K$ and $\operatorname{conv}(A)$ denotes the convex hull of $A$.

If $f: K \rightarrow \mathbb{R}$ is a convex continuous function, i.e., a continuous function such that

$$
f(\lambda x+(1-\lambda) y) \leq \lambda f(x)+(1-\lambda) f(y),
$$

then its maximum is attained in an extreme point $k_{0} \in K$. In fact, suppose that $k_{0} \in K$ is a point where the maximum is attained; the Minkowski/Krein-Milman Theorem asserts that there are $\lambda_{1}, \ldots, \lambda_{n} \in[0,1]$ such that

$$
k_{0}=\sum_{j=1}^{n} \lambda_{j} k_{j},
$$

with $k_{1}, \ldots, k_{n} \in \operatorname{ext} K$ and $\sum_{j=1}^{n} \lambda_{j}=1$. If the maximum of $f$ is not attained in any extreme point, then

$$
f\left(k_{0}\right) \leq \sum_{j=1}^{n} \lambda_{j} f\left(k_{j}\right)<\sum_{j=1}^{n} \lambda_{j} f\left(k_{0}\right)=f\left(k_{0}\right),
$$

a contradiction. The same happens with multilinear forms in finite dimensional normed spaces as the next result (which seems to be folklore) asserts:

Proposition 3. Let $m$ be a positive integer, $E$ be a finite dimensional normed space and $T: E \times \cdots \times E \rightarrow \mathbb{K}$ be an m-linear form. Then, denoting by $B_{E}$ its closed unit ball, we have

$$
\|T\|=\max \left\{\left|T\left(x_{1}, \ldots, x_{m}\right)\right|: x_{1}, \ldots, x_{m} \in \operatorname{ext} B_{E}\right\} .
$$

Proof. It suffices to prove that there are $y_{1}, \ldots, y_{m} \in \operatorname{ext} B_{E}$ such that

$$
\|T\|=\left|T\left(y_{1}, \ldots, y_{m}\right)\right|
$$

Let us suppose that (7) is not true. Since $B_{E}$ is compact, there exist $x_{1}, \ldots, x_{m} \in B_{E}$ such that $\left|T\left(x_{1}, \ldots, x_{m}\right)\right|=\|T\|$. By the Minkowski/Krein-Milman Theorem, we have

$$
B_{E}=\operatorname{conv}\left(\operatorname{ext}\left(B_{E}\right)\right)
$$

and thus

$$
x_{i}=\sum_{j_{i}=1}^{k_{i}} \lambda_{j_{i}}^{i} y_{j_{i}}^{i},
$$


with $\lambda_{j_{i}}^{i} \in[0,1], \sum_{j_{i}=1}^{k_{i}} \lambda_{j_{i}}=1$ and $y_{j_{i}}^{i} \in \operatorname{ext} B_{E}$ for all $i=1, \ldots, m$. Hence

$$
\begin{aligned}
\left|T\left(x_{1}, \ldots, x_{m}\right)\right| & =\left|T\left(\sum_{j_{1}=1}^{k_{1}} \lambda_{j_{1}}^{1} y_{j_{1}}^{1}, \ldots, \sum_{j_{m}=1}^{k_{m}} \lambda_{j_{n}}^{m} y_{j_{m}}^{m}\right)\right| \\
& \leq \sum_{j_{1}=1}^{k_{1}} \cdots \sum_{j_{m}=1}^{k_{m}} \lambda_{j_{1}}^{1} \cdots \lambda_{j_{m}}^{m}\left|T\left(y_{j_{1}}^{1}, \ldots, y_{j_{m}}^{m}\right)\right| \\
& <\|T\|,
\end{aligned}
$$

a contradiction.

The following lines illustrate how the previous results can be useful to our goals.

We want to solve the optimization problem

$$
\sup \left(\sum_{j_{1}, \ldots, j_{m}=1}^{n}\left|T\left(e_{j_{1}}, \ldots, e_{j_{m}}\right)\right|^{\frac{2 m}{m+1}}\right)^{\frac{m+1}{2 m}}
$$

for $T \in B_{\mathcal{L}\left({ }^{m} \ell_{\infty}^{n}(\mathbb{R})\right)}\left(\right.$ recall that $\mathcal{L}\left({ }^{m} \ell_{\infty}^{n}(\mathbb{R})\right)$ denotes the space of all $m$-linear forms $T: \ell_{\infty}^{n}(\mathbb{R}) \times \cdots \times$ $\ell_{\infty}^{n}(\mathbb{R}) \rightarrow \mathbb{R}$ and $B_{\mathcal{L}\left(m \ell_{\infty}^{n}(\mathbb{R})\right)}$ is its closed unit ball). Since $B_{\mathcal{L}\left(m \ell_{\infty}^{n}(\mathbb{R})\right)}$ is convex and compact and the function

$$
f: B_{\mathcal{L}\left(m \ell_{\infty}^{n}(\mathbb{R})\right)} \rightarrow \mathbb{R}
$$

given by

$$
f(T)=\left(\sum_{j_{1}, \ldots, j_{m}=1}^{n}\left|T\left(e_{j_{1}}, \ldots, e_{j_{m}}\right)\right|^{\frac{2 m}{m+1}}\right)^{\frac{m+1}{2 m}}
$$

is convex, the Minkowski/Krein-Milman Theorem tells us that the supremum of $f$ is attained in some extreme point of $B_{\mathcal{L}\left({ }^{m} \ell_{\infty}^{n}(\mathbb{R})\right)}$. Hence, we are guided to investigate the geometry of $B_{\mathcal{L}\left({ }^{m} \ell_{\infty}^{n}(\mathbb{R})\right)}$.

GEOMETRY OF UNIT BALL OF $\mathcal{L}\left({ }^{M} \ell_{\infty}^{N}(\mathbb{R})\right)$ : A MYSTERY TO BE SOLVED

The geometry of the unit ball of $\mathcal{L}\left({ }^{m} \ell_{\infty}^{n}(\mathbb{R})\right)$ is, in general, unknown. This section is entirely devoted to characterizing the geometry of the unit ball of $\mathcal{L}\left({ }^{2} \ell_{\infty}^{2}(\mathbb{R})\right)$. We start off with four elementary lemmata; the proofs are omitted.

Lemma 4. Let $a, b, c>0$ and $d<0$ be such that $a, b, c \geq-d$ and

$$
|a+b|+|c+d|=|a-b|+|c-d| .
$$

If $a \neq b$, then $b=-d$ or $a=-d$.

Lemma 5. Let $a, b, c>0$ and $d<0$. Then

$$
a+b+|c+d| \geq|a-b|+c-d
$$

if, and only if,

$$
a \geq-d, b \geq-d, c \geq-d \text { or } a \geq c, b \geq c,-d \geq c
$$


Lemma 6. Let $a, b, c>0$ and $d<0$. Then

$$
|a-b|+c-d \geq a+b+|c+d|
$$

if, and only if,

$$
a \geq b, c \geq b,-d \geq b \text { or } b \geq a, c \geq a,-d \geq a .
$$

Theorem 7. The extreme points of the closed unit ball of $\mathcal{L}\left({ }^{2} \ell_{\infty}^{2}(\mathbb{R})\right)$ are

$$
\begin{aligned}
& \pm x_{1} y_{1}, \pm x_{2} y_{1}, \pm x_{1} y_{2}, \pm x_{2} y_{2}, \\
& \frac{1}{2}\left( \pm x_{1} y_{1} \pm x_{2} y_{1} \pm x_{1} y_{2} \mp x_{2} y_{2}\right), \\
& \frac{1}{2}\left(\mp x_{1} y_{1} \pm x_{2} y_{1} \pm x_{1} y_{2} \pm x_{2} y_{2}\right), \\
& \frac{1}{2}\left( \pm x_{1} y_{1} \mp x_{2} y_{1} \pm x_{1} y_{2} \pm x_{2} y_{2}\right), \\
& \frac{1}{2}\left( \pm x_{1} y_{1} \pm x_{2} y_{1} \mp x_{1} y_{2} \pm x_{2} y_{2}\right) .
\end{aligned}
$$

Proof. For the sake of simplicity we shall denote $\ell_{\infty}^{2}(\mathbb{R})$ by $\ell_{\infty}^{2}$ along this proof. Let $T \in B_{\mathcal{L}\left({ }^{2} \ell_{\infty}^{2}\right)}$ be given by $T(x, y)=a x_{1} y_{1}+b x_{2} y_{1}+c x_{1} y_{2}+d x_{2} y_{2}$. By symmetry, it suffices to consider the following cases, with $a, b, c, d \neq 0$ :

(1) $T(x, y)=a x_{1} y_{1}$

(2) $T(x, y)=a x_{1} y_{1}+b x_{2} y_{1}$;

(3) $T(x, y)=a x_{1} y_{1}+b x_{2} y_{1}+c x_{1} y_{2}$;

(4) $T(x, y)=a x_{1} y_{1}+b x_{2} y_{1}+c x_{1} y_{2}+d x_{2} y_{2}$.

Since $T \in B_{\mathcal{L}\left({ }^{2} \ell_{\infty}^{2}\right)}$, we know that $|a|,|b|,|c|$ and $|d|$ are not bigger than 1 .

Case (1). If $|a|<1$, let $0<\varepsilon<1-|a|$. Defining

$$
\begin{aligned}
& A(x, y)=(a+\varepsilon) x_{1} y_{1} \\
& B(x, y)=(a-\varepsilon) x_{1} y_{1},
\end{aligned}
$$

we have $\frac{1}{2}(A+B)=T$ and $A, B \in B_{\mathcal{L}\left({ }^{2} \ell_{\infty}^{2}\right)}$. Thus, $T$ is not an extreme point. If $|a|=1$, we can suppose $a=1$. Thus, if there are $A, B \in B_{\mathcal{L}\left({ }^{2} \ell_{\infty}^{2}\right)}$ such that $\frac{1}{2}(A+B)=T$, say,

$$
\begin{gathered}
A(x, y)=\alpha x_{1} y_{1}+\beta x_{2} y_{1}+\gamma x_{1} y_{2}+\delta x_{2} y_{2} \\
B(x, y)=\alpha^{\prime} x_{1} y_{1}+\beta^{\prime} x_{2} y_{1}+\gamma^{\prime} x_{1} y_{2}+\delta^{\prime} x_{2} y_{2},
\end{gathered}
$$

we have $(\alpha, \beta, \gamma, \delta)=\left(2-\alpha^{\prime},-\beta^{\prime},-\gamma^{\prime},-\delta^{\prime}\right)$. Since $|\alpha|,\left|\alpha^{\prime}\right| \leq 1$, we conclude that $\alpha=\alpha^{\prime}=1$. Note that if $\beta \neq 0$, then $1+\beta$ or $1-\beta$ is bigger than 1 . Estimating $A((1,1),(1,0))$ and $A((1,1),(-1,0))$ we conclude that $\|A\|>1$ and the same happens to $B$; therefore $\beta=0$. The same argument shows us that $\gamma=\delta=0$. Thus, $T$ is an extreme point.

Case (2). Note that

$$
\|T\|=|a|+|b| \leq 1
$$

Let $0<\varepsilon<\min \{|a|,|b|\}$, and defining

$$
A(x, y)=(a+\operatorname{sgn}(a) \varepsilon) x_{1} y_{1}+(b-\operatorname{sgn}(b) \varepsilon) x_{2} y_{1}
$$




$$
B(x, y)=(a-\operatorname{sgn}(a) \varepsilon) x_{1} y_{1}+(b+\operatorname{sgn}(b) \varepsilon) x_{2} y_{1},
$$

we conclude that $\frac{1}{2}(A+B)=T$ and $A, B \in B_{\mathcal{L}\left({ }^{2} \ell_{\infty}^{2}\right)}$. Thus, $T$ is not an extreme point.

Case (3). By Proposition 2, we have

$$
\|T\|=\max \{|a+b|+|c|,|a-b|+|c|\} .
$$

Note that

$$
|a+b|+|c|=|a-b|+|c| \Leftrightarrow a=0 \text { or } b=0 .
$$

Let us consider two subcases:

(3A) $a b>0$;

(3B) $a b<0$.

If (3A) happens, then $|a-b|+|c|<1$. Defining $0<\varepsilon<\frac{1-(|a-b|+|c|)}{2}$ and

$$
\begin{aligned}
& A(x, y)=(a+\varepsilon) x_{1} y_{1}+(b-\varepsilon) x_{2} y_{1}+c x_{1} y_{2} \\
& B(x, y)=(a-\varepsilon) x_{1} y_{1}+(b+\varepsilon) x_{2} y_{1}+c x_{1} y_{2},
\end{aligned}
$$

we have $\frac{1}{2}(A+B)=T$ and $A, B \in B_{\mathcal{L}\left({ }^{2} \ell_{\infty}^{2}\right)}$. Thus $T$ is not extreme point.

If (3B) happens, then $|a+b|+|c|<1$. Defining $0<\varepsilon<\frac{1-(|a+b|+|c|)}{2}$ and

$$
\begin{aligned}
& A(x, y)=(a+\varepsilon) x_{1} y_{1}+(b+\varepsilon) x_{2} y_{1}+c x_{1} y_{2} \\
& B(x, y)=(a-\varepsilon) x_{1} y_{1}+(b-\varepsilon) x_{2} y_{1}+c x_{1} y_{2},
\end{aligned}
$$

we have $\frac{1}{2}(A+B)=T$ and $A, B \in B_{\mathcal{L}\left({ }^{2} \ell_{\infty}^{2}\right)}$. Thus $T$ is not extreme point.

Case (4). We consider four subcases:

(4A) $a b>0$ and $c d>0$;

(4B) $a b<0$ and $c d<0$;

(4C) $a b>0$ and $c d<0$;

(4D) $a b<0$ and $c d>0$.

If (4A) happens, then $|a-b|+|c-d|<1$. Considering $0<\varepsilon<\frac{1-(|a-b|+|c-d|)}{2}$ and defining

$$
\begin{aligned}
& A(x, y)=(a+\varepsilon) x_{1} y_{1}+(b-\varepsilon) x_{2} y_{1}+c x_{1} y_{2}+d x_{2} y_{2} \\
& B(x, y)=(a-\varepsilon) x_{1} y_{1}+(b+\varepsilon) x_{2} y_{1}+c x_{1} y_{2}+d x_{2} y_{2},
\end{aligned}
$$

we have $\frac{1}{2}(A+B)=T$ and $A, B \in B_{\mathcal{L}\left({ }^{2} \ell_{\infty}^{2}\right)}$. Thus $T$ is not an extreme point.

If (4B) happens, then $|a+b|+|c+d|<1$. Considering $0<\varepsilon<\frac{1-(|a+b|+|c+d|)}{2}$ and defining

$$
\begin{aligned}
& A(x, y)=(a+\varepsilon) x_{1} y_{1}+(b+\varepsilon) x_{2} y_{1}+c x_{1} y_{2}+d x_{2} y_{2} \\
& B(x, y)=(a-\varepsilon) x_{1} y_{1}+(b-\varepsilon) x_{2} y_{1}+c x_{1} y_{2}+d x_{2} y_{2},
\end{aligned}
$$

we have $\frac{1}{2}(A+B)=T$ and $A, B \in B_{\mathcal{L}\left({ }^{2} \ell_{\infty}^{2}\right)}$. Thus, again, $T$ is not an extreme point.

If (4C) happens we can assume $a, b, c>0$ and $d<0$. Note that by Proposition 2,

$$
\|T\|=|a+b|+|c+d|
$$


or

$$
\|T\|=|a-b|+|c-d| .
$$

We shall consider just (8) because (9) is similar. If we have (8) then, by Lemma 5 , there are two possibilities:

(4CA) $a \geq-d, b \geq-d$ and $c \geq-d$;

(4CB) $a \geq c, b \geq c$ and $-d \geq c$.

We shall first prove that if

$$
\operatorname{card}\{a, b, c,-d\} \neq 1,
$$

then $T$ is not an extreme point. Let us first suppose (4CA).

If $\operatorname{card}\{a, b, c,-d\} \neq 1$ we can assume $a \neq b$ because the other cases are analogous. We thus have two possibilities:

(4CAA) $a>b$,

(4CAB) $a<b$.

Let us first consider (4CAA):

Since $a>b$, we have $a>b \geq-d$ and $c \geq-d$. We consider two cases:

(4CAAA) $a>b>-d$ and $c \geq-d$;

(4CAAB) $a>b=-d$ and $c \geq-d$.

If (4CAAA) happens, since $a>b>-d>0$ and $c \geq-d>0$ we conclude that

$$
a+b+c+d>a-b+c-d
$$

i.e.,

$$
|a+b|+|c+d|>|a-b|+|c-d|
$$

and thus, by Proposition 2, $|a-b|+|c-d|<1$. Considering $0<\varepsilon<\frac{1-(|a-b|+|c-d|)}{2}$ and defining

$$
\begin{aligned}
& A(x, y)=(a+\varepsilon) x_{1} y_{1}+(b-\varepsilon) x_{2} y_{1}+c x_{1} y_{2}+d x_{2} y_{2} \\
& B(x, y)=(a-\varepsilon) x_{1} y_{1}+(b+\varepsilon) x_{2} y_{1}+c x_{1} y_{2}+d x_{2} y_{2},
\end{aligned}
$$

we have $\frac{1}{2}(A+B)=T$ and $A, B \in B_{\mathcal{L}\left({ }^{2} \ell_{\infty}^{2}\right)}$. Thus $T$ is not an extreme point.

If (4CAAB) happens, since $b=-d$ and using that $a>b>0$ and $c \geq-d>0$ we have

$$
|a+b|+|c+d|=|a-b|+|c-d|=a+c
$$

and, by then by Proposition 2,

$$
\|T\|=a+c \leq 1
$$

We have two possibilities:

(4CAABA) $a+c<1$;

(4CAABB) $a+c=1$.

If (4CAABA) happens, we choose $0<\varepsilon<\min \{a-b, 1-(a+c)\}$ and define

$$
\begin{aligned}
& A(x, y)=(a+\varepsilon) x_{1} y_{1}+b x_{2} y_{1}+c x_{1} y_{2}-b x_{2} y_{2} \\
& B(x, y)=(a-\varepsilon) x_{1} y_{1}+b x_{2} y_{1}+c x_{1} y_{2}-b x_{2} y_{2},
\end{aligned}
$$


and by Lemma 4, we conclude that $A, B \in B_{\mathcal{L}\left({ }^{2} \ell_{\infty}^{2}\right)}$ and $\frac{1}{2}(A+B)=T$. Hence, once more, $T$ is not an extreme point.

If (4CAABB) happens, we can write

$$
T(x, y)=a x_{1} y_{1}+b x_{2} y_{1}+(1-a) x_{1} y_{2}-b x_{2} y_{2} .
$$

Since $c \geq-d$, it follows that

$$
1-a \geq b
$$

If $1-a=b$, then

$$
T(x, y)=(1-b) x_{1} y_{1}+b x_{2} y_{1}+b x_{1} y_{2}-b x_{2} y_{2}
$$

Note that

$$
|(1-b)-b|+|b-(-b)|=|1-2 b|+2 b .
$$

Since $1-b=a>b>0$, it follows that $0<b<\frac{1}{2}$. Considering $0<\varepsilon<\min \left\{b, \frac{a-b}{2}\right\}$ and defining

$$
\begin{aligned}
& A(x, y)=(1-b+\varepsilon) x_{1} y_{1}+(b-\varepsilon) x_{2} y_{1}+(b-\varepsilon) x_{1} y_{2}+(-b+\varepsilon) x_{2} y_{2} \\
& B(x, y)=(1-b-\varepsilon) x_{1} y_{1}+(b+\varepsilon) x_{2} y_{1}+(b+\varepsilon) x_{1} y_{2}+(-b-\varepsilon) x_{2} y_{2},
\end{aligned}
$$

we have $\frac{1}{2}(A+B)=T$ and $\|A\|=\|B\|=1$. Hence, $T$ is not an extreme point.

If $1-a>b$, then $b<a<1-b$. Considering $0<\varepsilon<\min \{a-b, 1-a-b\}$ and defining

$$
\begin{aligned}
& A(x, y)=(a+\varepsilon) x_{1} y_{1}+b x_{2} y_{1}+(1-a-\varepsilon) x_{1} y_{2}-b x_{2} y_{2} \\
& B(x, y)=(a-\varepsilon) x_{1} y_{1}+b x_{2} y_{1}+(1-a+\varepsilon) x_{1} y_{2}-b x_{2} y_{2},
\end{aligned}
$$

we conclude that $A, B \in B_{\mathcal{L}\left({ }^{2} \ell_{\infty}^{2}\right)}$ and $\frac{1}{2}(A+B)=T$. Thus $T$ is not a extreme point.

Now let us prove (4CAB). Since $b>a$, then $b>a \geq-d$ and $c \geq-d$.

If $b>a>-d$ and $c \geq-d$, then

$$
a>-d \Rightarrow a+d>-a-d \Rightarrow a+b+c+d>b-a+c-d .
$$

Hence

$$
|a+b|+|c+d|>|a-b|+|c-d| .
$$

Considering $0<\varepsilon<\frac{1-(|a-b|+|c-d|)}{2}$ and defining

$$
\begin{aligned}
& A(x, y)=(a+\varepsilon) x_{1} y_{1}+(b-\varepsilon) x_{2} y_{1}+c x_{1} y_{2}+d x_{2} y_{2} \\
& B(x, y)=(a-\varepsilon) x_{1} y_{1}+(b+\varepsilon) x_{2} y_{1}+c x_{1} y_{2}+d x_{2} y_{2},
\end{aligned}
$$

we conclude that $\frac{1}{2}(A+B)=T$ and $A, B \in B_{\mathcal{L}\left({ }^{2} \ell_{\infty}^{2}\right)}$. Thus $T$ is not an extreme point.

If $b>a=-d$ and $c \geq-d$, then we shall proceed as in the case (4CAAB) to observe that

$$
T(x, y)=a x_{1} y_{1}+b x_{2} y_{1}+c x_{1} y_{2}-a x_{2} y_{2}
$$

is not an extreme point.

So, it remains to look for extreme points in the case (4C) when

$$
\operatorname{card}\{a, b, c,-d\}=1 .
$$


In this case we can write

$$
T(x, y)=a x_{1} y_{1}+a x_{2} y_{1}+a x_{1} y_{2}-a x_{2} y_{2} .
$$

Since $2 a=\|T\| \leq 1$, we have $a \leq \frac{1}{2}$. If $a<\frac{1}{2}, T$ is not an extreme point. Let us show that when $a=\frac{1}{2}$ the bilinear form $T$ given by (10) is an extreme point.

Suppose that there exist $A, B \in B_{\mathcal{L}\left({ }^{2} \ell_{\infty}^{2}\right)}$ such that $\frac{1}{2}(A+B)=T$. Denoting

$$
\begin{gathered}
A(x, y)=\alpha x_{1} y_{1}+\beta x_{2} y_{1}+\gamma x_{1} y_{2}+\delta x_{2} y_{2}, \\
B(x, y)=\alpha^{\prime} x_{1} y_{1}+\beta^{\prime} x_{2} y_{1}+\gamma^{\prime} x_{1} y_{2}+\delta^{\prime} x_{2} y_{2},
\end{gathered}
$$

we have

$$
\left(\alpha+\alpha^{\prime}, \beta+\beta^{\prime}, \gamma+\gamma^{\prime}, \delta+\delta^{\prime}\right)=(1,1,1,-1) .
$$

Since $|\alpha|,\left|\alpha^{\prime}\right| \leq 1$, it follows that $\alpha \in[0,1]$. A similar argument tells us that $\beta, \gamma,-\delta \in[0,1]$. We claim that if $\alpha \neq \frac{1}{2}$, then $A \notin B_{\mathcal{L}\left({ }^{2} \ell_{\infty}^{2}(\mathbb{R})\right)}$ or $B \notin B_{\mathcal{L}\left({ }^{2} \ell_{\infty}^{2}(\mathbb{R})\right)}$. Note that

$$
\begin{aligned}
0 \leq \alpha<\frac{1}{2} & \Rightarrow-\frac{1}{2}<-\alpha \leq 0 \\
& \Rightarrow \frac{1}{2}<1-\alpha \leq 1 \\
& \Rightarrow \frac{1}{2}<\alpha^{\prime} \leq 1
\end{aligned}
$$

and

In a similar fashion,

$$
\frac{1}{2}<\alpha \leq 1 \Rightarrow 0 \leq \alpha^{\prime}<\frac{1}{2}
$$

$$
0 \leq \beta<\frac{1}{2} \Rightarrow \frac{1}{2}<\beta^{\prime} \leq 1
$$

and so on.

So, let us first suppose $\alpha \in\left[0, \frac{1}{2}\right)$. We may have $\beta \in\left[0, \frac{1}{2}\right]$ or $\beta \in\left(\frac{1}{2}, 1\right]$.

If $\beta \in\left[0, \frac{1}{2}\right]$, then $\alpha^{\prime} \in\left(\frac{1}{2}, 1\right]$ and $\beta^{\prime} \in\left[\frac{1}{2}, 1\right]$. Therefore,

$$
B((1,1),(1,0))=\alpha^{\prime}+\beta^{\prime}>1
$$

and thus $\|B\|>1$, a contradiction.

If $\beta \in\left(\frac{1}{2}, 1\right]$, then we may have:

(P1) $\gamma \in\left[0, \frac{1}{2}\right]$ and $\delta \in\left[-1,-\frac{1}{2}\right]$;

(P2) $\gamma \in\left[0, \frac{1}{2}\right]$ and $\delta \in\left(-\frac{1}{2}, 0\right]$;

(P3) $\gamma \in\left(\frac{1}{2}, 1\right]$ and $\delta \in\left[-1,-\frac{1}{2}\right]$;

(P4) $\gamma \in\left(\frac{1}{2}, 1\right]$ and $\delta \in\left(-\frac{1}{2}, 0\right]$.

If (P1) holds, then $\alpha^{\prime} \in\left(\frac{1}{2}, 1\right], \gamma^{\prime} \in\left[\frac{1}{2}, 1\right]$ and $\beta^{\prime},-\delta^{\prime} \in\left[0, \frac{1}{2}\right)$. Thus $\alpha^{\prime}>\beta^{\prime}, \gamma^{\prime} \geq \beta^{\prime}$ and $\alpha^{\prime}, \gamma^{\prime} \geq-\delta^{\prime}$. When $\beta^{\prime} \geq-\delta^{\prime}$, by Lemma 5, we have

$$
\|B\|=\alpha^{\prime}+\beta^{\prime}+\gamma^{\prime}+\delta^{\prime}>1
$$

When $-\delta^{\prime} \geq \beta^{\prime}$, by Lemma 6 , we have

$$
\|B\|=\alpha^{\prime}-\beta^{\prime}+\gamma^{\prime}-\delta^{\prime}>1 .
$$


If (P2) holds, then $\alpha^{\prime},-\delta^{\prime} \in\left(\frac{1}{2}, 1\right], \gamma^{\prime} \in\left[\frac{1}{2}, 1\right]$ and $\beta^{\prime} \in\left[0, \frac{1}{2}\right)$. Thus $\alpha^{\prime},-\delta^{\prime}>\beta^{\prime}$ and $\gamma^{\prime} \geq \beta^{\prime}$. By Lemma 6, we have

$$
\|B\|=\alpha^{\prime}-\beta^{\prime}+\gamma^{\prime}-\delta^{\prime}>1
$$

If (P3) holds, then

$$
A((1,-1),(0,1))=-\delta+\gamma>1
$$

and thus $\|A\|>1$.

If we have (P4), then $\beta, \gamma \geq \alpha$ and $\beta, \gamma \geq-\delta$. When $\alpha \geq-\delta$, by Lemma 5, we have

$$
\|A\|=\alpha+\beta+\gamma+\delta>1
$$

When $-\delta \geq \alpha$, by Lemma 6 , we have

$$
\|A\|=\beta-\alpha+\gamma-\delta>1
$$

Now, let us suppose $\alpha \in\left(\frac{1}{2}, 1\right]$. We may have $\beta \in\left[0, \frac{1}{2}\right]$ or $\beta \in\left[\frac{1}{2}, 1\right]$. If $\beta \in\left[\frac{1}{2}, 1\right]$, then

$$
A((1,1),(1,0))=\alpha+\beta>1
$$

and hence $\|A\|>1$, a contradiction. If $\beta \in\left[0, \frac{1}{2}\right]$, we may have:

(K1) $\gamma \in\left[0, \frac{1}{2}\right]$ and $\delta \in\left[-1,-\frac{1}{2}\right]$;

(K2) $\gamma \in\left[0, \frac{1}{2}\right]$ and $\delta \in\left(-\frac{1}{2}, 0\right]$;

(K3) $\gamma \in\left(\frac{1}{2}, 1\right]$ and $\delta \in\left[-1,-\frac{1}{2}\right]$;

(K4) $\gamma \in\left(\frac{1}{2}, 1\right]$ and $\delta \in\left(-\frac{1}{2}, 0\right]$.

If (K1) happens, then $\alpha>\beta,-\delta \geq \beta$ and $\alpha,-\delta \geq \gamma$. When $\beta \geq \gamma$, by Lemma 5, we have

$$
\|A\|=\alpha+\beta-\delta-\gamma>1 .
$$

When $\gamma \geq \beta$, by Lemma 6 , we have

$$
\|A\|=\alpha-\beta+\gamma-\delta>1 .
$$

If (K2) occurs, then $\gamma^{\prime} \in\left[\frac{1}{2}, 1\right] \mathrm{e}-\delta^{\prime} \in\left(\frac{1}{2}, 1\right]$. Therefore,

$$
B((1,-1),(0,1))=-\delta^{\prime}+\gamma^{\prime}>1
$$

and $\|B\|>1$.

If we have (K3), then

$$
A((1,-1),(0,1))=-\delta+\gamma>1
$$

and so $\|A\|>1$.

If (K4) happens, then $\alpha, \gamma \geq \beta$ and $\alpha, \gamma \geq-\delta$. When $\beta \geq-\delta$, by Lemma 5, we have

$$
\|A\|=\alpha+\beta+\gamma+\delta>1
$$

When $-\delta \geq \beta$, by Lemma 6 , we have

$$
\|A\|=\alpha-\beta+\gamma-\delta>1 .
$$

The case (4CB) is analogous to (4CA) and (4D) is similar to (4C). 
Using the above characterization of extreme points and the Minkowski/Krein-Milman Theorem we have an alternative proof that the optimal constants of the Littlewood's $4 / 3$ inequality is $\sqrt{2}$. This geometric approach will be later commented in the final section.

Remark 8. Theorem 7 was independently proved in Kim (In Press).

\section{CONNECTIONS WITH LINEAR ABSOLUTELY SUMMING OPERATORS}

The aim of this section is to highlight the connections between the Bohnenblust-Hille/Hardy-Littlewood inequalities and the theory of absolutely summing operators. We show how recent results of the theory of Bohnenblust-Hille and Hardy-Littlewood inequalities are connected to classical results of the linear theory of absolutely summing operators. The results are essentially applications of recent contributions from Pellegrino et al. (In Press).

Let $E, F$ be Banach spaces and $r \geq s \geq 1$ be real numbers; let $E^{*}$ denote the topological dual of $E$ and $B_{E^{*}}$ be the closed unit ball of $E^{*}$. For all $p>1$, the real number $p^{*}$ denotes its conjugate number, i.e.,

$$
\frac{1}{p}+\frac{1}{p^{*}}=1 \text {. }
$$

We recall that a continuous linear operator $T: E \rightarrow F$ is absolutely $(r, s)$-summing if $\left(T\left(x_{j}\right)\right)_{j=1}^{\infty} \in \ell_{r}(F)$ whenever $\left(x_{j}\right)_{j=1}^{\infty} \in \ell_{s}^{w}(E)$, where

$$
\ell_{s}^{w}(E):=\left\{\left(x_{j}\right)_{j=1}^{\infty} \subset E: \sum_{j=1}^{\infty}\left|\varphi\left(x_{j}\right)\right|^{s}<\infty \text { for all } \varphi \in E^{*}\right\},
$$

which is a Banach space when endowed with the norm

$$
\left\|\left(x_{j}\right)_{j=1}^{\infty}\right\|_{w, s}=\sup _{\varphi \in B_{E^{*}}}\left(\sum_{j=1}^{\infty}\left|\varphi\left(x_{j}\right)\right|^{s}\right)^{1 / s} .
$$

The next result seems to be folklore.

Proposition 9. The following assertions are equivalent for $1 \leq p \leq q<\infty$ :

(i) The canonical inclusion map inc $: \ell_{p} \rightarrow \ell_{q}$ is absolutely $(r ; s)$-summing with constant $C$.

(ii)

$$
\left(\sum_{i=1}^{\infty}\left(\sum_{j=1}^{\infty}\left|A\left(e_{i}, e_{j}\right)\right|^{q}\right)^{\frac{1}{q} r}\right)^{\frac{1}{r}} \leq C\|A\|
$$

for all continuous bilinear forms $A: \ell_{s^{*}} \times \ell_{p^{*}} \rightarrow \mathbb{K}$.

Proof. (ii) $\Rightarrow\left(\right.$ i). Let $\left(x_{j}\right)_{j=1}^{\infty} \in \ell_{s}^{w}\left(\ell_{p}\right)$. According to Diestel et al. (1995) there is an isometric isomorphism $\Psi: \mathcal{L}\left(\ell_{s^{*}} ; \ell_{p}\right) \rightarrow \ell_{s}^{w}\left(\ell_{p}\right)$ given by $\Psi(v)=\left(v\left(e_{j}\right)\right)_{j=1}^{\infty}$; so there is a continuous linear operator $v: \ell_{s^{*}} \rightarrow$ $\ell_{p}=\left(\ell_{p^{*}}\right)^{*}$ such that $v\left(e_{j}\right)=x_{j}$ for all $j$. Then

$$
\left(\sum_{j=1}^{\infty}\left\|x_{j}\right\|_{\ell_{q}}^{r}\right)^{\frac{1}{r}}=\left(\sum_{j=1}^{\infty}\left(\sum_{i=1}^{\infty}\left|v\left(e_{j}\right)\left(e_{i}\right)\right|^{q}\right)^{\frac{1}{q} r}\right)^{\frac{1}{r}} .
$$


Recalling that $\mathcal{L}\left(\ell_{s^{*}}, \ell_{p^{*}} ; \mathbb{K}\right)=\mathcal{L}\left(\ell_{s^{*}} ; \ell_{p}\right)$ isometrically, there is a bilinear form $A \in \mathcal{L}\left(\ell_{s^{*}}, \ell_{p^{*}} ; \mathbb{K}\right)$ such that

$$
\left(\sum_{j=1}^{\infty}\left\|x_{j}\right\|_{\ell_{q}}^{r}\right)^{\frac{1}{r}}=\left(\sum_{j=1}^{\infty}\left(\sum_{i=1}^{\infty}\left|A\left(e_{j}, e_{i}\right)\right|^{q}\right)^{\frac{1}{q} r}\right)^{\frac{1}{r}} \leq C\|A\|=C\|v\|=\left\|\left(x_{j}\right)_{j=1}^{\infty}\right\|_{w, s} .
$$

(i) $\Rightarrow$ (ii). It is just to use the same isometric isomorphisms in the inverse direction.

In Pellegrino et al. (In Press) it was proved that if $q, r>0$ and $u, v \geq 2$ are such that

$$
\frac{1}{u}+\frac{1}{v}<1
$$

then

$$
\left(\sum_{i=1}^{\infty}\left(\sum_{j=1}^{\infty}\left|A\left(e_{i}, e_{j}\right)\right|^{q}\right)^{\frac{1}{q} r} \leq C\|A\|\right.
$$

for all continuous bilinear forms $A: \ell_{u} \times \ell_{v} \rightarrow \mathbb{K}$ if, and only if,

$$
\left\{\begin{array}{c}
q \geq \frac{v}{v-1} \\
r \geq \frac{u v}{u v-u-v} \\
\frac{1}{q}+\frac{1}{r} \leq \frac{3}{2}-\left(\frac{1}{u}+\frac{1}{v}\right) .
\end{array}\right.
$$

It is also well known that if

$$
\frac{1}{u}+\frac{1}{v} \geq 1
$$

then then the inequality (11) is always impossible. So, by Proposition 9 we conclude that if $1 \leq p, s \leq 2$, then inc: $\ell_{p} \rightarrow \ell_{q}$ (with $p \leq q$ of course) is absolutely $(r ; s)$-summing if and only if

$$
\left\{\begin{array}{c}
q \geq \frac{p^{*}}{p^{*}-1}=p \\
r \geq \frac{p^{*} s^{*}}{p^{*} s^{*}-p^{*}-s^{*}}=\frac{p s}{p+s-p s} \\
\frac{1}{q}+\frac{1}{r} \leq \frac{3}{2}-\left(\frac{1}{u}+\frac{1}{v}\right)=\frac{3}{2}-\left(\frac{1}{p^{*}}+\frac{1}{s^{*}}\right), \\
\frac{1}{s^{*}}+\frac{1}{p^{*}}<1, \text { i.e., } s<p^{*},
\end{array}\right.
$$

i.e.,

$$
\left\{\begin{array}{c}
r \geq \max \left\{\frac{2 p q s}{2 q s-2 p s+2 p q-p q s}, \frac{p s}{p+s-p s}\right\}, \\
s<p^{*} .
\end{array}\right.
$$

We thus recover the following inequalities of Bennett (1977) regarding inclusion summing norms: 
Theorem 10. Consider the canonical inclusion inc $: \ell_{p} \rightarrow \ell_{q}$ (with $p \leq q$ of course). If $1 \leq p, s \leq 2$, it is absolutely $(r ; s)$-summing if, and only if,

$$
\begin{aligned}
& r \geq \max \left\{\frac{2 p q s}{2 q s-2 p s+2 p q-p q s}, \frac{p s}{p+s-p s}\right\}, \\
& s<p^{*} .
\end{aligned}
$$

When $s=1$ we recover results of Bennett (1973):

Theorem 11. Consider the inclusion inc $: \ell_{p} \rightarrow \ell_{q}$ (with $p \leq q$ of course). If $1 \leq p \leq 2$ it is absolutely $(r ; 1)$-summing if and only if

$$
\begin{aligned}
& r \geq p \text { when } q \leq 2 \\
& r \geq \frac{2 p q}{2 q-2 p+p q} \text { when } q \geq 2 .
\end{aligned}
$$

Remark 12. Our results also imply that when $p=s=1$ the optimal value of the constant for the case of real scalars is $\sqrt{2}$.

\section{CONCLUSIONS}

Despite the progresses obtained in the last years there seems to be a long way until the Bohnenblust-Hille and Hardy-Littlewood inequalities are fully understood. In view of the analytic difficulties, a computational approach is reasonable alternative. In fact, some attempts in this direction can be seen in Araujo et al. (2017) and Cavalcante et al. (2016), but these approaches are restricted to very particular cases. A recent result of Pellegrino and Teixeira (2017) shows that a particular instance of the Bohnenblust-Hille inequality can be re-written as an algorithm, opening some possibilities for further computational investigations.

Since 2012, the main advances in the estimates of the constants of the Bohnenblust-Hille and HardyLittlewood inequalities were mainly obtained by interpolation, i.e., a kind of Hölder-type inequality for mixed $\ell_{p}$ spaces. However, as remarked in Pellegrino and Teixeira (2017) this seems to be still a sub-optimal approach. Several evidences were collected to support this claim and the notions of entropy and complexity introduced in Pellegrino and Teixeira (2017) may be helpful in this direction.

It is our belief that the geometry of the unit ball of $\mathcal{L}\left({ }^{m} \ell_{\infty}^{n}(\mathbb{R})\right)$, although very hard and delicate, should be investigated in depth and an eventual advance in this direction, combined with the Minkowski/KreinMilman Theorem could be an effective approach. Similarly, the investigation of the extreme points of the closed unit ball of $\mathcal{L}\left({ }^{m} \ell_{p}^{n}(\mathbb{R})\right)$ seems to be the best option to provide a final answer to the questions related to the optimal constants of the Hardy-Littlewood inequalities.

\section{ACKNOWLEDGMENTS}

The authors thank the two anonymous referees for important suggestions. W.V. Cavalcante is supported by Coordenação de Aperfeiçoamento de Pessoal de Nível Superior (CAPES) and D.M. Pellegrino is supported by Conselho Nacional de Desenvolvimento Científico e Tecnológico (CNPq) - Brasil, Grant 302834/2013-3. 


\section{REFERENCES}

ALBUQUERQUE N, ARAUJO G, MAIA M, NOGUEIRA T AND SANTOS J. 2017. Optimal Hardy-Littlewood inequalities uniformly bounded by a universal constant. arXiv: 1609.03081.

ALBUQUERQUE N, BAYART F, PELLEGRINO D AND SEOANE-SEPÚLVEDA J. 2014. Sharp generalizations of the multilinear Bohnenblust-Hille inequality. J Funct Anal 266: 3726-3740.

ARAUJO G, JIMÉNEZ-RODRÍGUEZ P, MUÑOZ-FERNÁNDEZ G AND SEOANE-SEPÚLVEDA J. 2017. Equivalent norms in polynomial spaces and applications. J Math Anal Appl 445: 1200-1220.

ARAUJO G AND PELLEGRINO D. 2014. Lower bounds for the constants of the Hardy-Littlewood inequalities. Linear Algebra Appl 463: 10-15.

ARAUJO G AND PELLEGRINO D. 2017. On the constants of the Bohnenblust-Hille and Hardy-Littlewood inequalities. Bull Braz Math Soc 48: 141-169.

BAYART F, PELLEGRINO D AND SEOANE-SEPÚLVEDA J. 2014. The Bohr radius of the $n$-dimensional polydisc is equivalent to $\sqrt{(\log n) / n}$. Adv Math 264: 726-746.

BENNETT G. 1973. Inclusion mappings between $l_{p}$ spaces. J Functional Anal 13: 20-27.

BENNETT G. 1977. Schur multipliers. Duke Math J 44: 603-639.

BOHNENBLUST H AND HILLE E. 1931. On the absolute convergence of Dirichlet series. Ann Math 32: 600-622.

BOHR H. 1913. Über die gleichmäßige Konvergenz Dirichletscher Reihen. Zeitschrift für reine und angewandte Mathematik, Bd 143.

CAMPOS J, JIMÉNEZ-RODRÍGUEZ P, MUÑOZ-FERNÁNDEZ G, PELLEGRINO D AND SEOANE-SEPÚLVEDA J. 2015. On the real polynomial Bohnenblust-Hille inequality. Lin Algebra Appl 465: 391-400.

CAVALCANTE W, NÚÑEZ-ALARCÓN D AND PELLEGRINO D. 2016. New lower bounds for the constants in the real polynomial Hardy-Littlewood inequality. Numer Funct Anal Optim 37: 927-937.

DEFANT A, FRERICK L, ORTEGA-CERDÀ J, OUNAÏES M AND SEIP K. 2011. The Bohnenblust-Hille inequality for homogeneous polynomials is hypercontractive. Ann Math 174: 485-497.

DIESTEL J, JARCHOW H AND TONGE A. 1995. Absolutely summing operators. Cambridge Studies in Advanced Mathematics 43.

DIMANT V AND SEVILLA-PERIS P. 2016. Summation of coefficients of polynomials on $\ell_{p}$ spaces. Publ Mat 60: 289-310.

DINIZ D, MUÑOZ-FERNÁNDEZ G, PELLEGRINO D AND SEOANE-SEPÚLVEDA J. 2014. Lower bounds for the constants in the Bohnenblust-Hille inequality: the case of real scalars. Proc Amer Math Soc 142: 575-580.

HARDY GH AND LITTLEWOOD JE. 1934. Bilinear forms bounded in space $[p, q]$. Quart J Math 5: 241-254.

JAMESON GJO. 1994. A specific form of Grothendieck's inequality for the two-dimensional case, with applications to C*-algebras. Proc Edinburgh Math Soc 37: 521-537.

KIM SG. IN PRESS. The geometry of $\mathcal{L}\left({ }^{2} l_{\infty}^{2}\right)$. Kyungpook Math J.

LITTLEWOOD JE. 1930. On bounded bilinear forms in an infinite number of variables. Quart J Math 1: 164-174.

MAIA M, NOGUEIRA T AND PELLEGRINO D. 2017. The Bohnenblust-Hille inequality for polynomials whose monomials have a uniformly bounded number of variables, to appear in Integral Equations and Operators Theory. DOI: 10.1007/ s00020-017-2372-z.

MONTANARO A. 2012. Some applications of hypercontractive inequalities in quantum information theory. J Math Phys 53: 15.

NUNES AG. 2017. A new estimate for the constants of an inequality due to Hardy and Littlewood. Linear Algebra Appl 526: 27-34.

PELLEGRINO D, SANTOS J, SERRANO-RODRÍGUEZ D AND TEIXEIRA E. IN PRESS. Regularity principle in sequence spaces and applications. Bull Sci Math.

PELLEGRINO D AND TEIXEIRA E. 2017. Towards sharp Bohnenblust-Hille constants, to appear in Comm Contemp Math. DOI: http://dx.doi.org/10.1142/S0219199717500298.

PRACIANO-PEREIRA T. 1981. On bounded multilinear forms on a class of $\ell_{p}$ spaces. J Mat Anal Appl 81: 561-568. 